\title{
Prevalence of Dermatophytosis and Antifungal Activity of Ethanolic Crude Leaf Extract of Tetradenia riparia against Dermatophytes Isolated from Patients Attending Kampala International University Teaching Hospital, Uganda
}

\author{
Taufik Kakande, ${ }^{1}$ Yonah Batunge, ${ }^{2}$ Emmanuel Eilu, ${ }^{3}$ Ambrose Shabohurira, ${ }^{3}$ \\ Justus Abimana $\left(\mathbb{D},{ }^{3}\right.$ Saheed Adekunle Akinola, ${ }^{3}$ Reagan Muhwezi, ${ }^{3}$ \\ Abubakar Sunusi Adam, ${ }^{3}$ Sarah Kemuma Onkoba, ${ }^{3}$ Adamu Almustapha Aliero $\mathbb{D}^{3}$, \\ Collins Atuheire $\mathbb{D}^{4},{ }^{4}$ Charles Drago Kato, ${ }^{3,5}$ and Ibrahim Ntulume $\mathbb{D}^{3}$ \\ ${ }^{1}$ School of Pharmacy, Kampala International University Western Campus, Ishaka, P.O. Box 71, Bushenyi, Uganda \\ ${ }^{2}$ Department of Medical Laboratory Science, Kampala International University Western Campus, Ishaka, \\ P.O. Box 71, Bushenyi, Uganda \\ ${ }^{3}$ Department of Microbiology and Immunology, Faculty of Biomedical Sciences, Kampala International University Western Campus, \\ Ishaka, P.O. Box 71, Bushenyi, Uganda \\ ${ }^{4}$ Department of Public Health, Kampala International University Teaching Hospital, P.O. Box 71, Bushenyi, Uganda \\ ${ }^{5}$ School of Bio-Security, Biotechnical and Laboratory Sciences, College of Veterinary Medicine, Animal Resources and Biosecurity \\ (COVAB), Makerere University, P.O. Box 7062, Kampala, Uganda
}

Correspondence should be addressed to Ibrahim Ntulume; ntulumeibrahim@kiu.ac.ug

Received 24 January 2019; Revised 24 April 2019; Accepted 15 May 2019; Published 11 July 2019

Academic Editor: Craig G. Burkhart

Copyright (C) 2019 Taufik Kakande et al. This is an open access article distributed under the Creative Commons Attribution License, which permits unrestricted use, distribution, and reproduction in any medium, provided the original work is properly cited.

Dermatophyte infections are a global health problem but neglected in Uganda. This work aimed at determining prevalence of dermatophytosis and antifungal activity of ethanolic crude leaf extract of Tetradenia riparia against dermatophytes isolated from patients attending Kampala International University Teaching Hospital (KIU-TH), Uganda. A total of 100 samples of skin and nail scrapings were collected and processed using standard microscopy $(\mathrm{KOH})$ and cultural methods. T. riparia leaves were collected and processed with 95\% ethanol using standard extraction method. The crude leaves ethanolic extract was tested against three dermatophytes: Trichophyton tonsurans, T. mentagrophyte, and Microsporum audouinii using modified agar well diffusion method. Minimum inhibitory concentration (MIC) and minimum fungicidal concentration (MFC) of the ethanolic leaves crude extract were also determined using broth tube dilution and culture, respectively. Out of 100 samples collected, 49 (49\%, 95\%CI: 0.3930-0.5876) were found positive for microscopy. The prevalence of dermatophytosis was significantly $(\mathrm{p}=0.001)$ associated with age groups of participants with higher infection among those aged 11-20 and 21-30 years with 75.0\% each. Out of the 49 that were positive by microscopy, 28 (57.15\%, 95\% CI: 0.1987-0.3739) were positive by culture. Thirty-one (31) fungal isolates were obtained which included both dermatophyte and non-dermatophyte fungi. T. verrucosum had highest distribution 6 (19.35\%) among dermatophytes species while Aspergillus spp. were found to have highest distribution 7 (22.58\%) among non-dermatophyte species. The result of the antidermatophytic test showed that T. riparia ethanolic crude leaves extract had activity against tested dermatophytes at $1 \mathrm{~g} / \mathrm{ml}$. MIC and MFC of the crude extract of T. riparia against tested dermatophytes ranged from 62.5 to $250 \mathrm{mg} / \mathrm{ml} \mathrm{and} 125$ to $500 \mathrm{mg} / \mathrm{ml}$, respectively. The findings of this study reported the presence of dermatophytes causing dermatophytosis among patients attending KIU-TH. The results of the current study showed that T. riparia leaves ethanolic crude extract has antidermatophytic activity against tested dermatophytes. 


\section{Introduction}

Dermatophytes are a group of fungi that invade keratinized tissues of the hair, skin (epidermis), and nails, and some cause conditions commonly referred to as Tinea-meaning group of disease. They grow best in humid and warm environment hence common in the subtropical and humid regions. Recent classifications of dermatophytes using multilocus phylogenetic study regroup the organisms into seven (7) genera: Arthroderma, Epidermophyton, Lophophyton, Microsporum, Nannizzia, Paraphyton, and Trichophyton [1]. The most causative organisms of tinea capitis fall in three genera: Trichophyton, Microsporum, and Nannizzia [1]. Tinea infection can spread from person to person (anthropophilic) or indirectly from fomites (e.g., clothes hair brush and huts), through soil (geophilic) or contact with animals (zoophilic) [2]. They infect areas of the body such as the groin (Tinea cruris or "jock itch"), hands/legs, full body (Tinea corporis or "ringworm"), hips, waist, scalp (Tinea capitis or "scalp ringworm"), and nails (Tinea unguium or onychomycosis), respectively [2-4]. As reviewed by Bongomin et al. [5], the global burden of cutaneous infection was estimated to be $\sim 1001000,000$. These infections are more common among rural than urban population; the infections are more pronounced in males as compared to females and high in children especially Tinea capitis [6]. Literature has shown that the global burden of dermatophytic infection specifically was estimated to be $20-25 \%$ [7]. There was scarcity of data on dermatophytic infection in Uganda especially from western part of the country, but T. capitis affects $10 \%$ of all Kenyan school children [7, 8]. Wiegand et al. [9] reported $82.6 \%$ prevalence of dermatophytosis in children attending Mbarara Regional Referral Hospital in Uganda. Dermatophyte infections are therefore a serious but neglected health problem in Uganda [10]. Several species of Trichophyton and Microsporum have been isolated from tinea capitis ("scalp ringworm") lesions [10]. The predominant infecting species is T. tonsurans. Disease manifestations range from small scaling patches to involvement of the entire scalp with extreme hair loss [10]. Although T. capitis infections are not life threatening, they have significant social and health related economic impacts $[5,11]$ due to reoccurrences from exposure to the sources of the causative organisms such as sharing towels, clothing, or hair accessories with infected individuals [8], long duration of treatment regimens, poor adherence, and cases of reoccurrences [12].

Although many effective antifungal drugs are available, most of them are relatively expensive [13] and others with marked toxic effects such as hepatotoxicity. Plants used in herbal treatment as antifungal agents such as T. riparia [14] could provide an alternative, relatively cheap, and improved accessibility to treatment for such opportunistic infections especially in HIV/AIDS.

T. riparia or misty plum bush (English) is a medicinal plant also locally known as kyewamala (Luganda), Akachuchu (Rotoro), and Omuravunga (Runyankole). It is used locally for the treatment of a variety of illnesses such as malaria, several skin diseases (fungi, cleaning wounds, itching, body rashes, and scabies), ulcers, headache, bilharzias, worms, rheumatism, emetic, dyspepsia, fever, colds, cough, toothache, psychotic excitements, and blocked fallopian tubes [15]. In Uganda, T. riparia plant was reported to be used in treatment of both bacterial and fungal infection [14]. Therefore, this study was aimed at determining the prevalence of dermatophytosis and antifungal activity of ethanolic crude leaf extract of T. riparia against dermatophytes isolated from patients attending Kampala International University Teaching Hospital, Uganda.

\section{Materials and Methods}

2.1. Study Area and Study Design. This was a laboratory experimental study involving isolation and identification of dermatophytes causing fungi from patients attending Community HIV-AIDS Initiative (CHAI) and Dermatology Clinic at Kampala International University Teaching Hospital, Western Campus, Uganda. Isolation and identification of dermatophytosis causing fungi were done at Mycology Laboratory, Department of Microbiology, Mycology Unit, Mbarara Regional Referral Hospital. Plant samples (leaves) were collected from Rukararwe Bushenyi District $\left(0^{\circ} 31^{\prime} 27.0^{\prime \prime} \mathrm{S} 30^{\circ} 13^{\prime} 02.1^{\prime \prime} \mathrm{E}\right)$ [16]. Extraction, phytochemical screening, and antidermatophytic activity testing were carried out at the Microbiology and Immunology and Pharmacology Laboratories at Department of Microbiology and Immunology and School of Pharmacy Kampala International University, Western Campus.

2.2. Inclusion and Exclusion Criteria. All patients that attended CHAI and Dermatology Clinic of KIU-TH presenting with skin diseases, who consented or their parents assented on their behalf in case of patients below 18 years old, were included in this study. Patients that attended CHAI and Dermatology Clinic of KIU-TH presenting with signs and symptoms of dermatophytosis and did not consent were excluded. Patients who were on antifungal treatment were not also included in this study.

2.3. Sample Collection. One hundred (100) samples of infected skin, nail, and scalp craping were collected from selected study participants attending Community HIV-AIDS Initiative (CHAI) and Dermatology Clinic through the help of a volunteer who is registered health personnel (medical laboratory personnel). Samples were collected according to the method described by Taha et al. [17] with little modification. The sites of infections were first cleaned with surgical spirit, and scales from the skin lesions were collected by scraping outwards with a blunt scalpel from the edge of the lesion. Specimens from the scalp and nail were collected using forceps by plucking the specimen from the scalp and nail. All samples were collected on a sterile piece of paper $(5 \mathrm{~cm}$ square). The papers were folded to enclose the specimen, labeled, and transferred to Mycology Laboratory, Department of Microbiology, Mycology Unit, Mbarara Regional Referral Hospital, for isolation and identification. 
2.4. Isolation and Identification of Tests Organisms. Samples collected were subjected to direct microscopic examination after immersing them in $10 \% \mathrm{KOH}$ for skin scrapings or hair for ten minutes and $20 \% \mathrm{KOH}$ for nail for twenty minutes and viewed under microscope using X10 lower magnification and confirmed using X40 higher magnification for the presence of hyphae and arthroconidia, respectively [18]. Scrapings of scalp nail and skin samples were inoculated on the freshly prepared Sabourad Dextrose Agar (SDA) containing chloramphenicol at a concentration of $0.5 \mathrm{mg} / \mathrm{ml}$ and $0.4 \mathrm{~g} / \mathrm{l}$ of cycloheximide in order to inhibit the growth of bacteria and saprophytic fungi, respectively. The inoculated plates were incubated at $25^{\circ} \mathrm{C}$ for up to 21 days. Suspected colony of dermatophytes was subcultured to freshly prepared SDA media to obtain pure cultures. The suspected colonies of dermatophytes and molds were identified to species level based on macroscopy (colony color, pigment production, topography, and texture) and microscopic morphology such as small unicellular microconidia and larger septate macroconidia using Lactophenol cotton blue mount [19]. Candida spp. were identified also using phenotypic methods of chromogenic media and germ tube test.

2.5. Plant Samples Collection. Plant sample collection, identification, and drying were done according to the method described by Esazah [14]. The fresh leaves of $T$. riparia were collected from Rukararwe, Bushenyi District. The plants materials were taken for identification to a Botanist at Mbarara University of Science and Technology. The plants materials were identified using Martin the Kew database available at www.theplantlist.org [14]. The leaves were dried under shade to avoid decomposition of volatile chemical constituents. The dried leaves were powdered using mortar and pestle, then sieved into fine powder, and stored in airtight jar.

2.6. Extraction of Ethanolic Crude Extract. Extraction of the ethanolic crude extract was carried out according the method described by Esazah [14] with some modification. Two hundred grams $(200 \mathrm{~g})$ of powder was soaked in ethanol $(95 \% \mathrm{v} / \mathrm{v})$ in a two-liter (2 l) conical flask, with periodic shaking for $24 \mathrm{~h}$. The extract was filtered off using Whatman No. 1 filter paper and concentrated (ethanol left to evaporate off) in hot air oven at $45^{\circ} \mathrm{C}$. The concentrated crude extracts were kept at $4^{\circ} \mathrm{C}$ in a refrigerator in a container and labelled appropriately. The yield $(\%, w / w)$ from dried leaves crude extract was calculated using the following formula: yield (\%) $=\left(\mathrm{W}_{1} \times 100\right) / \mathrm{W}_{2}$, where $\mathrm{W}_{2}$ is the weight of powder before drying and $W_{1}$ is the weight of dry extract.

2.7. Phytochemical Screening. Phytochemical analysis of leaves crude ethanolic extract of $T$. riparia was done using standard procedures [20-22] with minor modification [23]. The parameters determined are tannins, phlobatannins, saponins, flavanoids, terpenoids, diterpenes, glycosides, alkaloids, steroids, and phenols.

2.8. Testing for Antidermatophytic Activity. The test organisms: T. tonsurans, T. mentagrophyte, and M. audouinii were aseptically inoculated at the center in different plates on freshly prepared sterile Sabouraud Dextrose Agar. Using sterile glass cork borers $(6 \mathrm{~mm}$ in diameter), 3 wells were carefully made on the agar plates without distorting the media at $0.05 \mathrm{~cm}$ distance away from the inoculated test organism. Two grams ( $2 \mathrm{~g}$ ) of $T$. riparia leaves crude extract was dissolved in $2 \mathrm{ml}$ of DMSO as stock solution [14]. Each well was filled with $100 \mu \mathrm{l}$ of $1 \mathrm{~g} / \mathrm{ml}$ extract and the other wells were filled with terbinafine $40 \mu \mathrm{g} / \mathrm{ml}$ as positive control and $10 \%$ DMSO as negative control, respectively [22]. The plates were then incubated at $27-30^{\circ} \mathrm{C}$ for 3-5 days [20]. The antidermatophytic activity was observed using dissecting microscope.

2.9. Minimum Inhibitory Concentration (MIC). T. tonsurans, T. mentagrophyte, and M. audouinii sporangial suspension concentration was adjusted to an optical density of 0.014 equivalent to $1.0 \times 10^{5}$ spores $/ \mathrm{ml}$ using a spectrophotometer at a wave length of $530 \mathrm{~nm}$ [20]. MIC of T. riparia leaves ethanolic crude extract was determined using tube dilution method. One milliliter $(1 \mathrm{ml})$ of Brain Heart infusion Broth $+0.5 \%$ Agar was placed in 10 tubes. One milliliter $(1 \mathrm{ml})$ of stock solution $2 \mathrm{~g} / \mathrm{ml}$ was transferred in the first test tube of each organism mixed and 2-fold serial dilution was done till concentrations below $0.0156 \mathrm{~g} / \mathrm{ml}$ were attained $[14,23]$. One milliliter $(1 \mathrm{ml})$ of positive control terbinafine $(2$ $\mathrm{mg} / \mathrm{ml}$ ) was transferred in different set of test tubes and also serially diluted (2-fold) using the same procedure as above. Then twenty microlitres $(20 \mu \mathrm{l})$ of inoculum preparation of each fungus was placed in the above serially dilluted tubes. One milliliter $(1 \mathrm{ml})$ of $10 \%$ DMSO was used as negative control. The test tubes were then incubated at 28 to $30^{\circ} \mathrm{C}$ for 4-7days. The test tubes were observed for the lowest concentration with no visible growth (no visual turbidity) and were considered as the MIC [23].

2.10. Determination of Minimum Fungicidal Concentration. Following the MIC determination using tube dilution method, MFC was determined by subculturing $20 \mu$ l of the culture from each negative well (with no visual turbidity) of both the extract and the positive control. MFC was defined as the lowest concentration resulting in negative subcultures (no growth) after incubation of the plates at 28 to $30^{\circ} \mathrm{C}$ for 3-5 days [20].

2.11. Ethical Consideration. Ethical approval was sought from the Kampala International University Research and Ethics Committee (KIU-REC). Permission to carry out the study was sought from the School of Allied Health Sciences, KIUWestern Campus. Participation in the study was on voluntary basis. Patients were enrolled in the study after consenting to the study following a verbal and written explanation of the study. For children, consent was provided by parents/legal guidance but assent was also requested from the children. All information was treated with utmost confidentiality.

2.12. Data Analysis. Results obtained were entered in Microsoft excel sheet. Descriptive analysis was used to obtain prevalence of dermatophytosis, mean and standard deviation 
of the age groups of the studied participants. Chi-square was used to compare between the prevalence of dermatophytosis and demographic characteristics of studied participants using SPSS software (version 16) and $\mathrm{p} \leq 0.05$ was considered significant.

\section{Results}

3.1. Demographic Characteristic of Studied Participants. One hundred (100) patients selected from patients attending CHAI and Dermatology Clinic of KIU-TH were enrolled in this study. Fifty-three (53\%) were males and 47 (47\%) were females. The age of the studied participants ranged from 1 to 69 years. The median age of study participants was 34.7 years.

3.2. Prevalence of Dermatophytosis. Out of 100 specimens collected, 49 (49 \%, 95\% CI: 0.3930-0.5876) were KOH positive. The prevalence of dermatophytosis based on microscopy according to gender showed that male participants had the highest prevalence $26 / 49$ (53.1\%) compared to their female counterpart $23 / 49$ (46.9\%). The gender of the participants was not significantly associated with prevalence of dermatophytosis with microscopic examination using chi-square test $(\mathrm{p}=0.990)$ (Table 1$)$. The prevalence of dermatophytosis among the studied participants according to their age groups based on microscopy showed that age groups of 11-20 and 2130 years had the highest prevalence with $75.0 \%$ each while age groups between 51 and 60 and $>60$ had the lowest prevalence with $10.00 \%$ each. However, the age group of the participants was statistically associated with the prevalence of dermatophytosis with microscopic examination using chisquare tests $(\mathrm{p}=0.001)$ (Table 2$)$.

Out of the 49 samples that were $\mathrm{KOH}$ positive, 28 (57.14\%, 95\%CI: 0.1987-0.3739) were culture positive. Prevalence of dermatophytosis based on culture showed that male had the highest prevalence $19 / 49(38.78 \%)$ as in Table 1 . The prevalence of dermatophytosis based on culture according to the age group of studied participants showed that age groups between 11 and 20 had the highest prevalence 10/49 (20.41\%) while age groups between 51 and 60 and $>60$ each had the lowest prevalence $0 / 49(0.00 \%)$ as in Table 2 .

3.3. Distribution of Dermatophytes and Non-Dermatophytes. All fungal isolates obtained were identified using standard colony morphological and microscopic characteristics (Table 3). Thirty-one fungal isolates were isolated from the 28 positive culture samples which included both dermatophytes (Figure 1) and non-dermatophytes fungi. Among the dermatophytes species, $T$. verrucosum was found to have the highest distribution 6 (19.35) in both gender and age of studied participants, while Aspergillus spp. were more distributed 7 (22.58) among non-dermatophytes species in both gender and age of studied participants as in Table 4 .

3.4. Percentage Yield and Qualitative Phytochemical Screening of $T$. riparia Ethanolic Crude Leaves Extract. The $T$. riparia ethanolic crude extract yielded $20.1 \mathrm{~g}$ of powder upon concentration giving a percentage yield of $10.05 \%$. The quantitative phytochemical screening of the $T$. riparia ethanolic leaves crude extract studied showed the presence of tannins, phenols, glycosides, and terpenoids and absence of saponins, flavanoids, phlobatannins, and anthraquinone (Table 5).

3.5. Antidermatophytic Susceptibility Assay of T. riparia Ethanolic Leaves Crude Extract. The antidermatophytic assay showed that $T$. riparia leaves crude extract and terbinafine (positive control) were active on all test dermatophytes ( $T$. tonsurans, T. mentagrophyte, and $M$. audouinii) studied at a concentration of $1 \mathrm{~g} / \mathrm{ml}$ and $40 \mu \mathrm{g} / \mathrm{ml}$, respectively. The negative control (10\% DMSO) showed no antidermatophytic activity (Figure 2). However, susceptibility of both terbinafine and extract varied among organisms with $T$. tonsurans being more susceptible followed by $T$. mentagrophyte while M. audouinii was less susceptible. Furthermore, terbinafine which was used as positive control showed higher antidermatophytic activity against all test dermatophytes compared to $T$. riparia leaves crude extract.

3.6. Minimum Inhibitory Concentration and Minimum Fungicidal Concentration. The MIC of the T. riparia leaves crude extract ranged from 62.5 to $250 \mathrm{mg} / \mathrm{ml}$. T. tonsurans had 62.5 $\mathrm{mg} / \mathrm{ml} \mathrm{MIC,} 125 \mathrm{mg} / \mathrm{ml}$ for T. mentagrophyte, and $250 \mathrm{mg} / \mathrm{ml}$ for M. audouinii. Terbinafine as positive control was effective at concentration of $0.0078 \mathrm{mg} / \mathrm{ml}$ against T. tonsurans, and 0.0078 and $0.0313 \mathrm{mg} / \mathrm{ml}$ against T. mentagrophyte and $M$. audouinii, respectively (Table 6).

The MFC value of the $T$. riparia leaves crude extract against all tested dermatophytes spp. ranged from 125 to 500 $\mathrm{mg} / \mathrm{ml}$. The MFC of the extract against T. tonsurans was 125 $\mathrm{mg} / \mathrm{ml}, 250 \mathrm{mg} / \mathrm{ml}$ for T. mentagrophyte, and $500 \mathrm{mg} / \mathrm{ml}$ for M. audouinii. MFC value of terbinafine ranged from 0.0156 to $0.0625 \mathrm{mg} / \mathrm{ml}$. Terbinafine was found to have MFC at concentration of $0.0156 \mathrm{mg} / \mathrm{ml}$ against $T$. tonsurans and 0.0313 $\mathrm{mg} / \mathrm{ml}$ and $0.0625 \mathrm{mg} / \mathrm{l}$ against $T$. mentagrophyte and $M$. audouinii, respectively. The low MFC values as those obtained with T. tonsurans and T. mentagrophyte denoted that these dermatophytes spp. are more susceptible to the antifungal agent while a high MFC value observed against $M$. audouinii denoted that this dermatophyte sp. was less susceptible to the antifungal agent (Table 6).

\section{Discussion}

Literatures have shown that fungal infections of the skin and scalp (dermatophytosis) represent a relatively common problem especially in the tropical and subtropical regions of the world where warm and humid climate provides a favourable environment for fungi [5, 24-26]. They have become a significant health problem affecting children, adolescents, and adults [27-29].

The result of the present study found the prevalence of dermatophytosis 49\% among patients attending CHAI and Dermatology Clinic at KIU-HT using microscopy $(\mathrm{KOH})$. The prevalence found in this study was lower compared to the prevalence $82.6 \%(n=115)$ reported by Wiegand et al. [9] in children attending Mbarara Regional Referral Hospital in Uganda using Blankophor. The lower prevalence found 


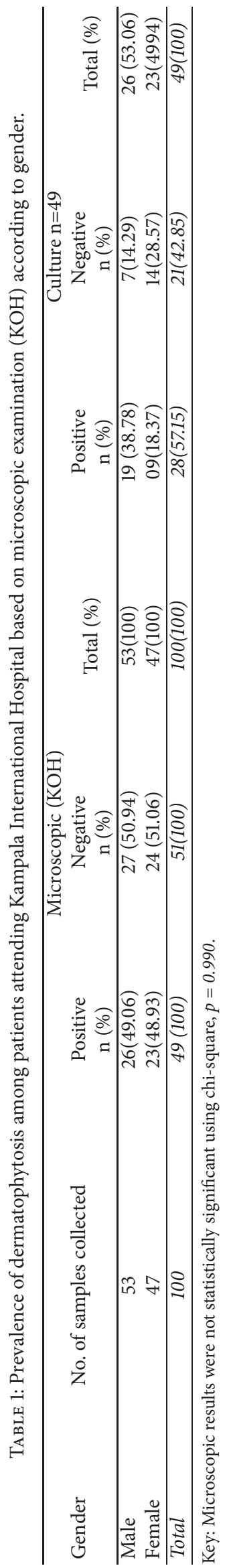


TABLE 2: Prevalence of dermatophytosis among patients attending Kampala International Hospital based on microscopic examination (KOH) according to age.

\begin{tabular}{|c|c|c|c|c|c|}
\hline \multirow[b]{2}{*}{ Age } & \multirow[b]{2}{*}{ No. of samples collected } & \multicolumn{2}{|c|}{ Microscopy (KOH) } & \multicolumn{2}{|c|}{ Culture $n=49$} \\
\hline & & Positive, n (\%) & $\begin{array}{c}\text { Negative } \\
\mathrm{n}(\%)\end{array}$ & Positive, n (\%) & $\begin{array}{c}\text { Negative } \\
\mathrm{n}(\%)\end{array}$ \\
\hline $1-10$ & 3 & $3(100)$ & $0(0.00)$ & $2(4.08)$ & $1(2.04)$ \\
\hline $11-20$ & 16 & $12(75.00)$ & $4(25.00)$ & $10(20.41)$ & $2(4.08)$ \\
\hline $21-30$ & 20 & $13(75.00)$ & $7(35.00)$ & $7(14.29)$ & $6(12.24)$ \\
\hline $31-40$ & 26 & $13(50.00)$ & $13(50.00)$ & $8(16.33)$ & $5(10.20)$ \\
\hline $41-50$ & 16 & $6(37.5)$ & $10(62.5)$ & $1(2.04)$ & $5(10.20)$ \\
\hline $51-60$ & 9 & $1(11.11)$ & $8(88.89)$ & $0(0.00)$ & $1(2.04)$ \\
\hline$>60$ & 10 & $1(10.00)$ & $9(90.00)$ & $0(0.00)$ & $1(2.04)$ \\
\hline Total & 100 & $\begin{array}{c}49(49) \\
95 \% \text { CI: } 0.3930-0.5876^{*}\end{array}$ & $51(51)$ & $\begin{array}{c}28(57.14) \\
95 \% C I: 0.1987-0.3739\end{array}$ & $21(42.86)$ \\
\hline
\end{tabular}

Key: Microscopic results were statistically significant for age group using chi-square, $p=0.001^{*}$, CI: confidence interval.

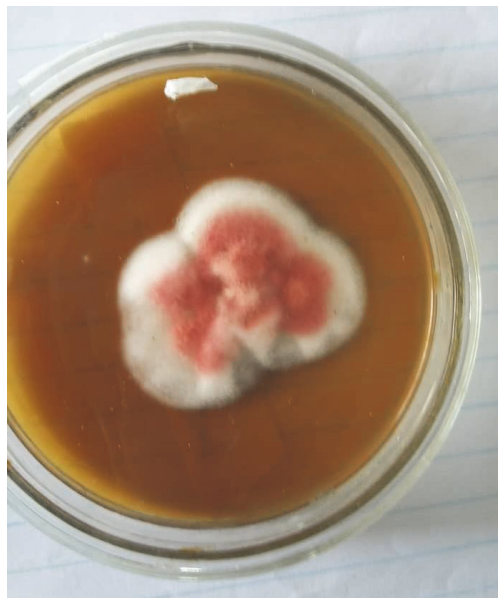

(a)

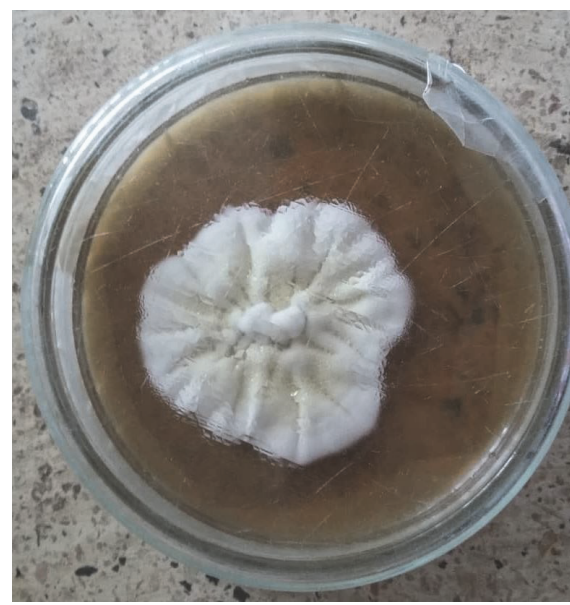

(b)

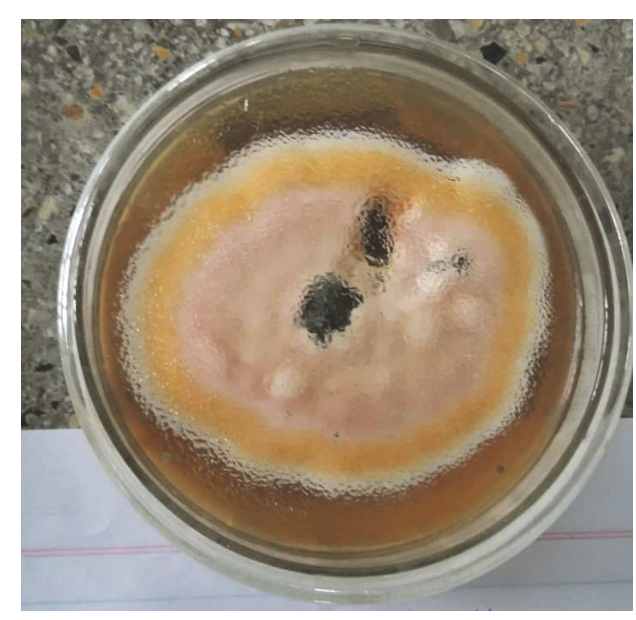

(d)

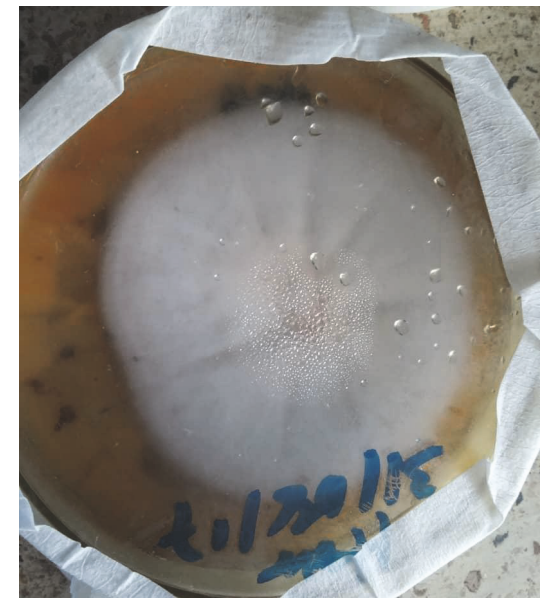

(c)

Figure 1: Colony morphology of some isolated dermatophytes species from patients attending Kampala International University Teaching Hospital, Uganda. (a) T. rubrum, (b) T. mentagrophytes, (c) M. audouinii, and (d) T. tonsurans. 


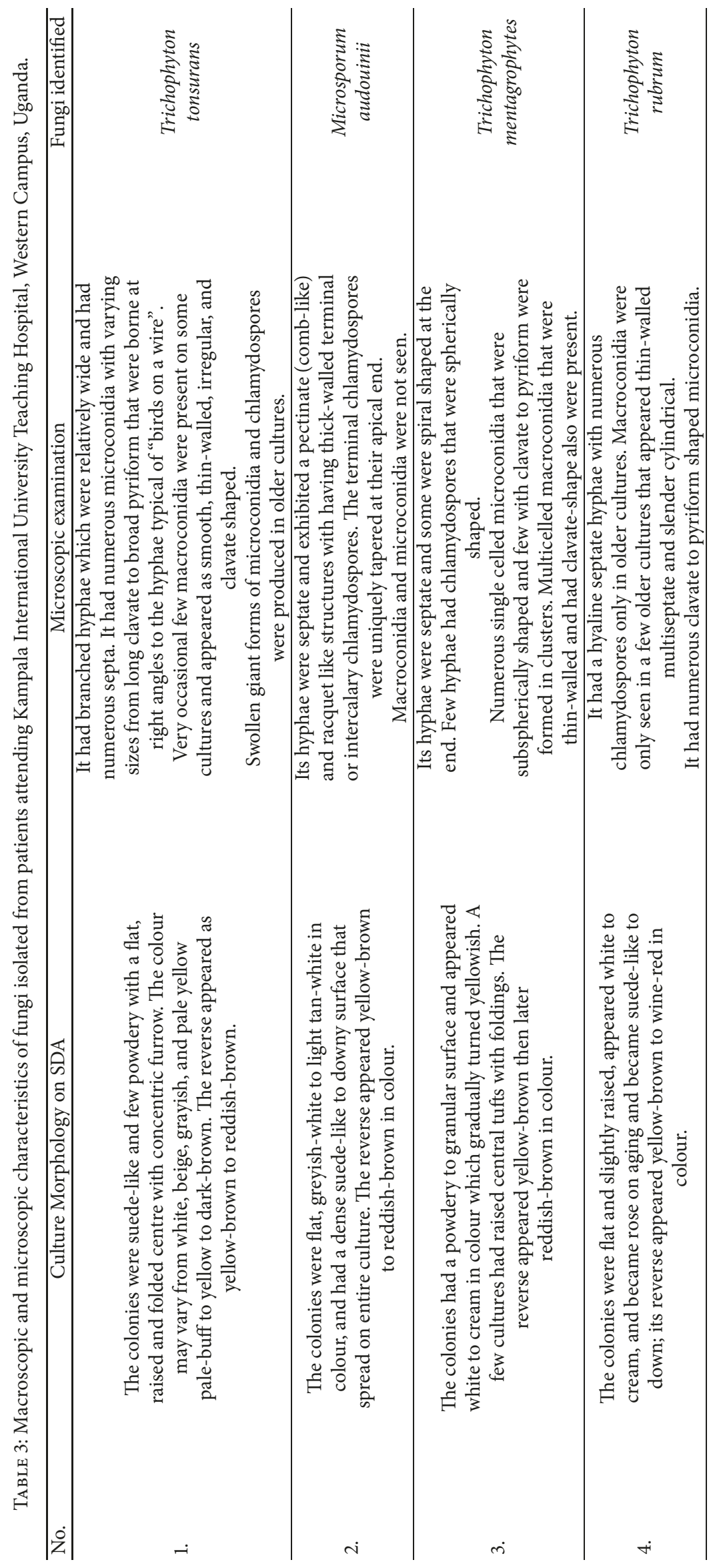




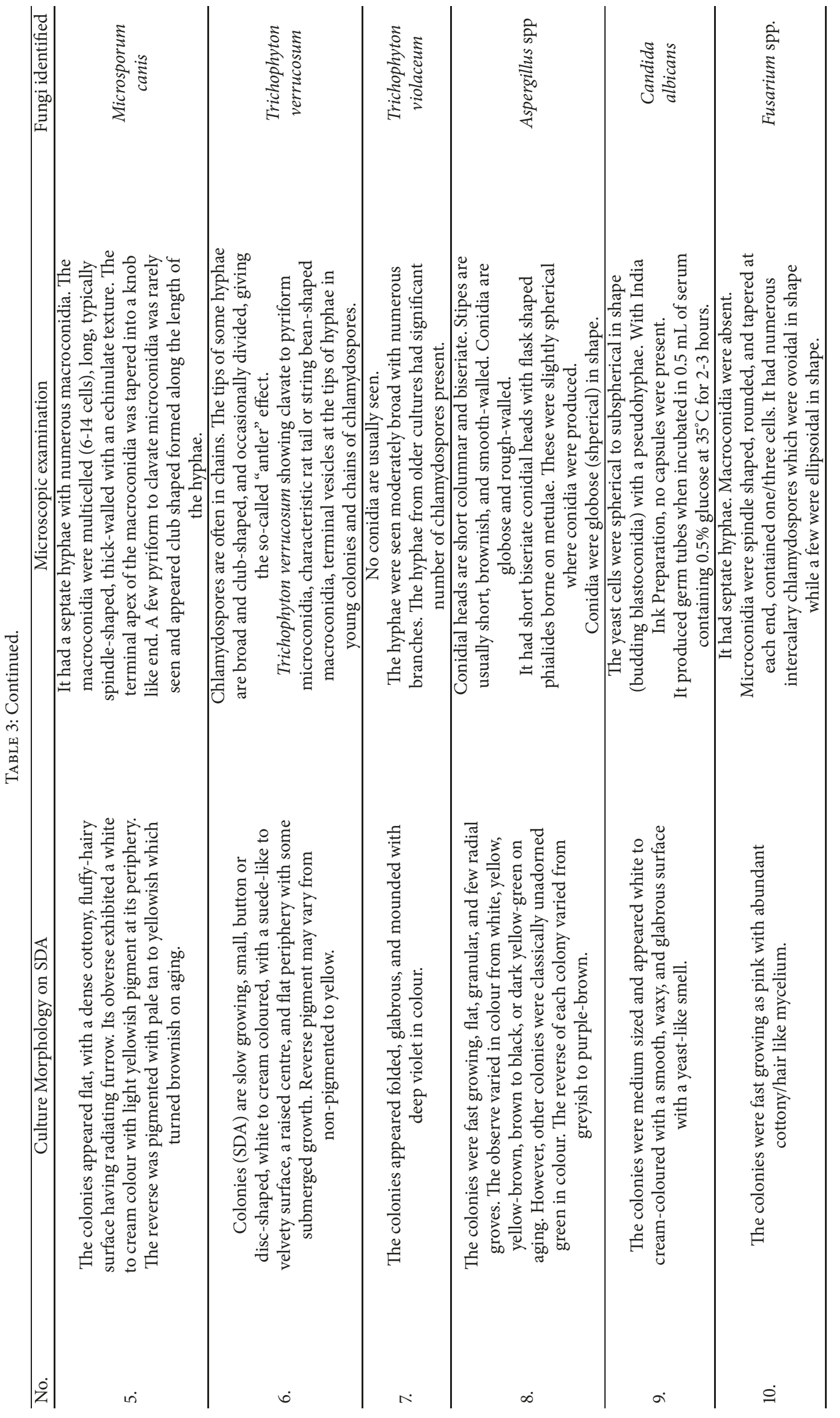




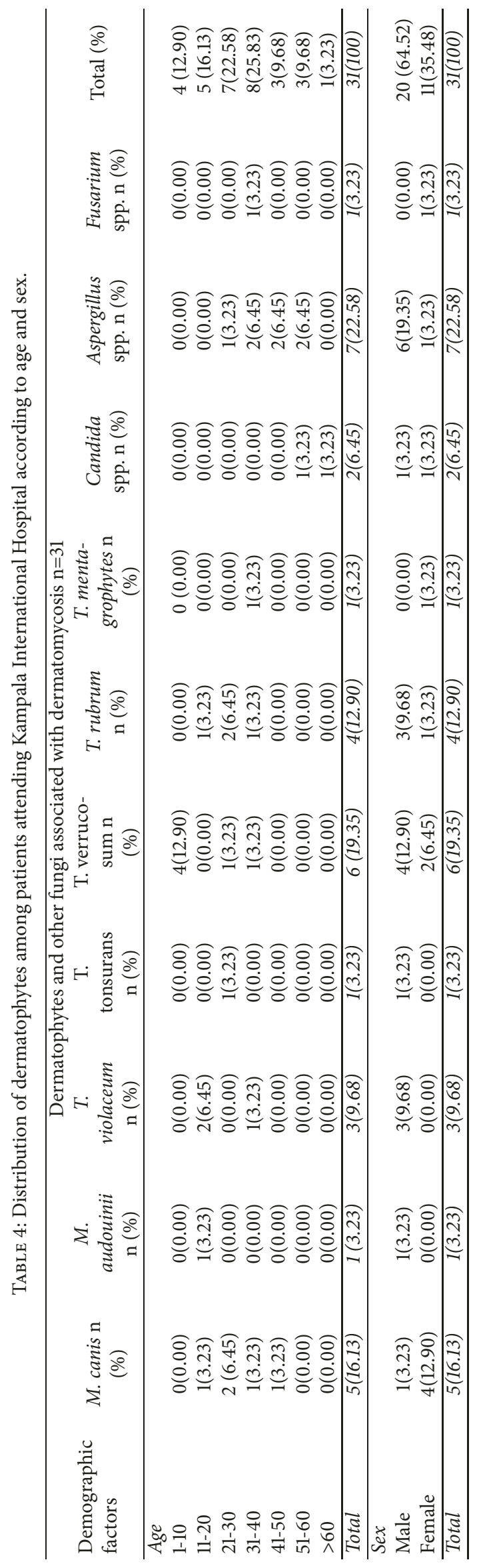


TABLE 5: Qualitative phytochemical screening of T. riparia ethanolic leaves crude extract.

\begin{tabular}{lcc}
\hline Phytochemical parameters & Inference \\
\hline Saponins & - \\
Tannins & + \\
Phenols & + \\
Flavanoids & - \\
Glycosides & + \\
Alkaloids & + \\
Steroids & + \\
Terpenoids & + \\
Phlobatannins & + \\
Anthraquinones & - \\
\hline
\end{tabular}

Key: (+) indicates presence and (-) indicates absence.

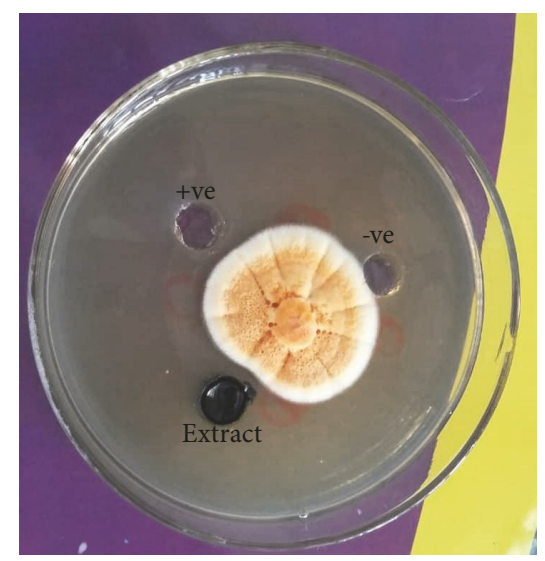

(a)

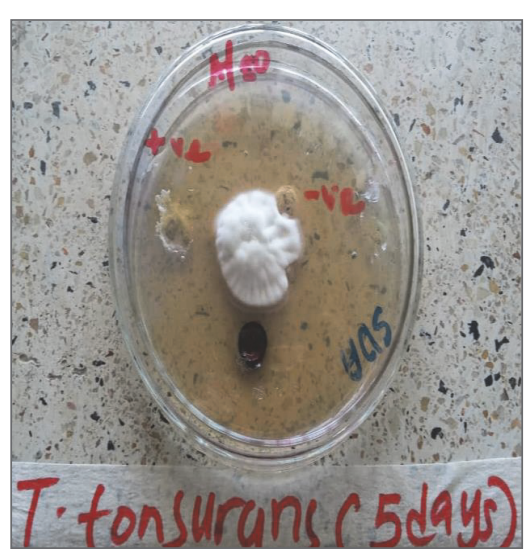

(b)

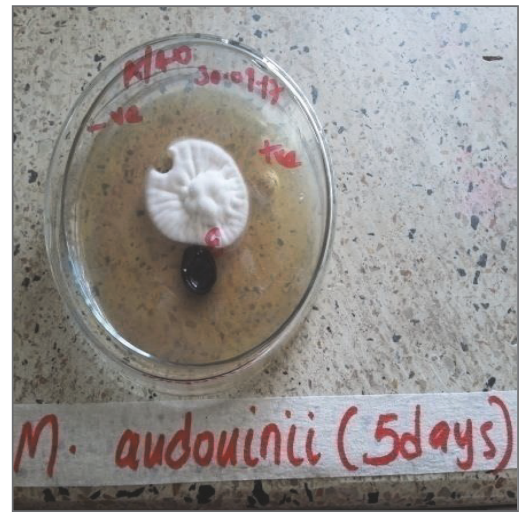

(c)

Figure 2: An antidermatophytic activity of Tetradenia riparia leaves crude extract, Terbinafine and \%10 DMSO against dermatophytes. (a) T. mentagrophyte, (b) T. tonsurans, and (c) M. audouinii. +ve: positive control, -ve: negative control.

in this study compared to Wiegand et al. [9] could be due to differences in studied participants where we used age groups between 1-67 years while in their study studied participants age groups were 1-16 years. Literature shows that dermatophytosis affects young children than the old $[5,6$, 25]. However, Moto et al. [8] reported prevalence of $68.0 \%$ $(n=150)$ in school going children from Mathare, informal settlement in Nairobi, Kenya. The prevalence reported in this study was higher than the prevalence $36.5 \%(n=236)$ and $45.0 \%(n=100)$ reported by Leiva-Salinas [29] and Dogo et al. [30] from Ethiopia and Nigeria, respectively.

Prevalence of dermatophytosis according to gender of studied participants found in this study showed that males have the highest prevalence compared to the females. This was in line with finding of Leiva-Salinas et al. [29] who reported higher prevalence of dermatophytosis in males than in females $42.2 \%$ and $30.5 \%$, respectively, among school children in a rural area in southern Ethiopia. This was contrary to the finding of Dogo et al. [30] who reported higher prevalence of dermatophytosis in girls than the boys $51.4 \%$ and $41.5 \%$, respectively, among school children in Nok community of Kaduna State, Nigeria.
The prevalence of dermatophytosis was significantly $(p=0.001)$ associated with age groups of studied participants which showed that age groups between 11-20 and 21-31 had the highest prevalence. This was in line with finding of Dogo et al. [30] who reported higher prevalence of dermatophytosis among age groups between 11 and 15 years among school children in Nok community of Kaduna State, Nigeria. This was contrary to the finding of Leiva-Salinas et al. [29] who reported higher prevalence of dermatophytosis $46.9 \%$ in age groups between 5 and 7 years among school children in a rural area in southern Ethiopia.

This study also revealed that out of the $49 \%$ samples that were positive by microscopy, only 28 samples were positive by culture. Wiegand et al. [9] reported $82.6 \%(\mathrm{n}=115)$ and $87.8 \%$ $(\mathrm{n}=101)$ for Blankophor and culture methods, respectively. Thirty-one fungal species were isolated from the 28 samples positive by culture method which involved dermatophytes and non-dermatophytes. Among the dermatophytes species, T. verrucosum was the most common agent of dermatophytosis in this study. This was contrary to the finding of Wiegand et al. [9] who reported higher distribution of T. violaceum $65.2(73 / 112)$ among the dermatophytes species isolated from 


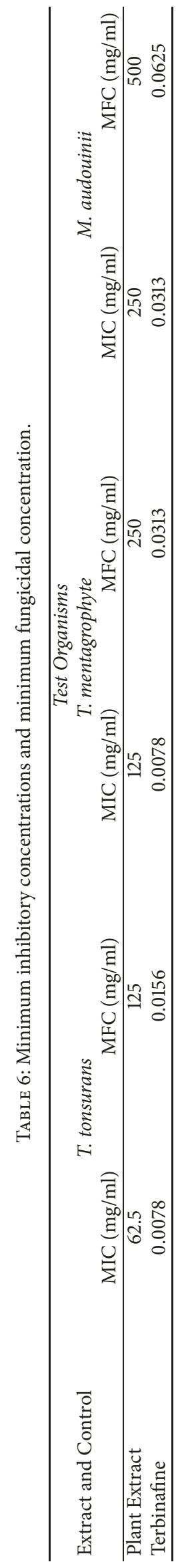


their study. However, Aspergillus spp. were found the most occurrent non-dermatophytes fungal species in this study. This was also contrary to the finding of Wiegand et al. [9] who reported higher prevalence of Scopulariopsis brevicaulis $6.3(7 / 112)$ from their study.

The percentage yield of $10.05 \%$ was obtained from $T$. riparia ethanolic leaves crude extract and was within the recommended range (10.96-20.29\%) according to the British Herbal Pharmacopoea [31]. This is also in line with the findings of [14].

Photochemistry of the plant revealed presence of tannins, steroids, glycosides, phenols, and terpenoids. This was similar to the finding of $[15,32]$ who reported the presence of reducing compounds, phenols and tannins from $T$. riparia ethanolic leaves crude extract. In addition, [33] reported the presence of saponins, flavonoids, and alkaloids from the T. riparia ethanolic leaves crude extract from Kenya. This confirmed the statement by [34] who reported that plant from different geographical area across the world may have different concentrations of the active substances.

Preliminary antifungal assay using modified agar well diffusion method revealed that $T$. riparia ethanolic leaves crude extract had antifungal activity against all tested dermatophytes at concentration of $1 \mathrm{~g} / \mathrm{ml}$, and terbinafine had antidermatophytic activity at a concentration of $40 \mu \mathrm{g} / \mathrm{ml}$. Endo et al. [35] also reported antidermatophytic activity of hydroalcoholic extract of $T$. riparia against some dermatophytes though they used disc diffusion method in their study. However, susceptibility of both terbinafine and extract varied among organisms with $T$. tonsurans being more susceptible followed by $T$. mentagrophyte while $M$. audouinii was less sensitive to the crude extract. The increased susceptibility to $T$. tonsurans and T. mentagrophyte could be attributed to their slow growth, i.e., T. tonsurans (8-12 days) and T. mentagrophyte (7 -10days) as compared to M. audouinii which grows in 5-10 days [33]; hence the antifungal agent arrests their initial stages of growth, hence limiting their ability to sporulate thus them having increased susceptibility. Furthermore, terbinafine which was used as positive control showed higher antidermatophytic activity against all tested dermatophytes spp. compared to $T$. riparia leaves crude extract. This could be due to the purity of the terbinafine compared to the T. riparia leaves crude extract which was in mixture form. Ahmad and Aqil [34] reported that extracts in crude forms may contain some compound that may have antagonistic activity against other bioactive compounds in the crude extracts leading to the low activity against tested organism.

The MIC and MFC of terbinafine were also generally less for T. tonsurans $(0.0078$ and $0.0156 \mathrm{mg} / \mathrm{ml})$, followed by $T$. mentagrophyte $(0.0078$ and $0.0313 \mathrm{mg} / \mathrm{ml})$ while $M$. audouinii had 0.033 and $0.0625 \mathrm{mg} / \mathrm{ml}$. This finding is similar to the findings of $[36,37]$ against similar organisms when using terbinafine. Terbinafine causes changes in the fungal cell permeability with consequent modification of the cell structure which also causes hyphae to become parched and wrinkled when viewed under an electron microscope. The variation in susceptibility patterns could be attributed to the growth period variations among the organisms [34].

\section{Conclusions}

The findings of this study reported the prevalence of dermatophytes causing dermatophytosis 49\% among patients attending Dermatology and CHAI Clinics of KIU-TH. The prevalence of dermatophytosis was significantly $(p=0.001)$ associated with age groups of studied participants. T. riparia leaves ethanolic crude extract contains bioactive compounds that are active against the tested dermatophytes (T. tonsurans, T. mentagrophyte, and M. audouinii). This supports its use in Western Ugandan traditional medicine for the treatment of skin diseases caused by dermatophytes and other fungal infections. This study found out the MIC range of the extract ranged from 62.5 to $250 \mathrm{mg} / \mathrm{ml}$ and MFC was 125 $500 \mathrm{mg} / \mathrm{ml}$. Further studies should be carried out on pure standard dermatophyte isolates included in the study and other dermatophyte and non-dermatophyte organisms not included in the study.

\section{Data Availability}

The [Tables and Figures] data used to support the findings of this study are included within the article.

\section{Conflicts of Interest}

The authors declare no conflicts of interest.

\section{Acknowledgments}

We would like to thank Mr. Muhwezi Jackson of Microbiology and Immunology Laboratory, Mr. Ibanda Ivan of Pharmacognosy Laboratory of Kampala International University Western Campus, and staff of Mycology Laboratory of Mbarara Regional Referral Hospital for their kind support during this research. We also thank the management of Kampala International University Teaching Hospital for granting us permission to carry out this study.

\section{References}

[1] G. S. de Hoog, K. Dukik, M. Monod et al., "Toward a Novel Multilocus Phylogenetic Taxonomy for the Dermatophytes," Mycopathologia, vol. 182, no. 1-2, pp. 5-31, 2017.

[2] R. R. Achterman and T. C. White, "Dermatophyte virulence factors: identifying and analyzing genes that may contribute to chronic or acute skin infections," International Journal of Microbiology, 2012.

[3] S. C. Gopinath, P. Anbu, T. Lakshmipriya et al., "Biotechnological aspects and perspective of microbial keratinase production," BioMed Research International, vol. 2015, Article ID 140726, 2015.

[4] R. Gupta and P. Ramnani, "Microbial keratinases and their prospective applications: an overview," Applied Microbiology and Biotechnology, vol. 70, no. 1, pp. 21-33, 2006.

[5] F. Bongomin, S. Gago, R. Oladele, and D. Denning, "Global and multi-national prevalence of fungal diseases-estimate precision," Journal of Fungi, vol. 3, no. 4, p. 57, 2017. 
[6] S. Vishnu, K. K. Tarun, S. Anima, S. Ruchi, and C. Subhash, "Dermatophytes: Diagnosis of dermatophytosis and its treatment," African Journal of Microbiology Research, vol. 9, no. 19, pp. 1286-1293, 2015.

[7] S. Neji, F. Makni, F. Cheikhrouhou et al., "Epidemiology of dermatophytoses in Sfax, Tunisia," Mycoses, vol. 52, no. 6, pp. 534-538, 2009.

[8] J. N. Moto, J. M. Maingi, and A. K. Nyamache, "Prevalence of Tinea capitis in school going children from Mathare, informal settlement in Nairobi, Kenya," BMC Research Notes, vol. 8, no. 1, p. 274, 2015.

[9] C. Wiegand, P. Mugisha, G. K. Mulyowa et al., "Identification of the causative dermatophyte of tinea capitis in children attending Mbarara Regional Referral Hospital in Uganda by PCR-ELISA and comparison with conventional mycological diagnostic methods," Medical Mycology, vol. 55, no. 6, pp. 660668, 2016.

[10] R. Parkes-Ratanshi, B. Achan, A. Kambugu, D. Meya, and D. Denning, "Estimated burden of fungal disease in Uganda," in Tropical Medicine \& International Health, vol. 12, pp. 929-935, 2007.

[11] L. Johnson, "Dermatophytes-the skin eaters," Mycologist, vol. 17, no. 4, pp. 147-149, 2003.

[12] M. O. H Uganda, "Fungal skin infections," in Uganda Clinical Guidelines, pp. 935-938, 2016.

[13] K. D. Smith, B. Achan, K. H. Hullsiek et al., "Increased Antifungal Drug Resistance in Clinical Isolates of Cryptococcus neoformans in Uganda," Antimicrobial Agents and Chemotherapy, vol. 59, no. 12, pp. 7197-7204, 2015.

[14] K. Esazah, A. Fredric, O. Jasper, and A. Godwin, "Phytochemical analysis and screening of ugandan medicinal plants for antifungal activity against candida albicans," International Journal of Tropical Disease \& Health, vol. 9, no. 1, pp. 1-8, 2015.

[15] N. I. de Melo, C. E. de Carvalho, L. Fracarolli et al., "Antimicrobial activity of the essential oil of Tetradenia riparia (Hochst.) Codd. (Lamiaceae) against cariogenic bacteria," Brazilian Journal of Microbiology, vol. 46, no. 2, pp. 519-525, 2015.

[16] Uganda National Meteorological Authority (UNMA), Weather and Climate for All, 2016, https://www.unma.go.ug/.

[17] M. Taha, M. Elfangary, S. Essa, and A. Younes, "Species identification of dermatophytes isolated from human superficial fungal infections by conventional and molecular methods," Journal of the Egyptian Women's Dermatologic Society, vol. 14, no. 2, pp. 76-84, 2017.

[18] J. Ndako, O. O. Osemwegie, T. H. Spencer, B. K. Olopade, G. A. Yunusa, and J. Banda, "Prevalence of dermatophytes and other associated fungi among school children," GARJMMS, vol. 1, no. 3, pp. 49-56, 2012.

[19] M. F. Mohamed Shalaby, A. Nasr El-din, and M. Abu El-Hamd, "Isolation, Identification, and In Vitro Antifungal Susceptibility Testing of Dermatophytes from Clinical Samples at Sohag University Hospital in Egypt," Electronic Physician, vol. 8, no. 6, pp. 2557-2567, 2016.

[20] P. Kumar, J. Kumar, R. Kumar, and R. C. Dubey, "Studies on phytochemical constituents and antimicrobial activities of leaves, fruits and stems of Solanum nigrum L," AJPSKY, vol. 6, no. 4, pp. 57-68, 2016.

[21] W. C. Evans and W. B. Saunder, Trease and Evans Pharmacognosy, WB Saunders Co. Ltd, 15th edition, 1989.

[22] L. Das and S. Godbole, "Antifungal and phytochemical analysis of lantana camara, citrus limonum (lemon, azadirachta indica (neem) and hibiscus rosasinensis (china rose)," Journal of Pharmacy Research, vol. 9, no. 7, pp. 476-479, 2015.

[23] S. Khan, S. Singhal, T. Mathur, D. Upadhyay, and A. Rattan, "Antifungal susceptibility testing method for resource constrained laboratories," Indian Journal of Medical Microbiology, vol. 24, no. 3, p. 171, 2006.

[24] S. A. Tsamiya, M. A. Usman, M. A. Seth et al., "Ring worm skin infection in a rural community of bushenyi (uganda)," British Microbiology Research Journal, vol. 9, no. 2, pp. 1-5, 2015.

[25] L. Ferguson and L. C. Fuller, "Spectrum and burden of dermatophytes in children," Infection, vol. 74, pp. S54-S60, 2017.

[26] D. M. Thappa, "Common skin problems in children," The Indian Journal of Pediatrics, vol. 69, no. 8, pp. 701-706, 2002.

[27] P. Kannan, C. Janaki, and G. S. Selvi, "Prevalence of dermatophytes and other fungal agents isolated from clinical samples," Indian Journal of Medical Microbiology, vol. 24, no. 3, pp. 212$215,2006$.

[28] A. Chepchirchir, C. Bii, and J. Ndinya-Achola, "Dermatophyte Infections In Primary School Children In Kibera Slums Of Nairobi," East African Medical Journal, vol. 86, no. 2, 2009.

[29] M. Leiva-Salinas, I. Marin-Cabanas, I. Betlloch et al., "Tinea capitis in schoolchildren in a rural area in southern Ethiopia," International Journal of Dermatology, vol. 54, no. 7, pp. 800-805, 2015.

[30] J. Dogo, S. L. Afegbua, and E. C. Dung, "Prevalence of Tinea capitis among school children in Nok community of Kaduna state," Prevalence of Tinea capitis among school children in Nok community of Kaduna state, pp. 10-1155, 2016.

[31] British Herbal pharmacopoeia, "British herbal association," ISBN, Article ID 903032, 1996.

[32] M. H. Ndiku and M. C. Ngule, "Antibacterial activity and Preliminary phytochemical screening of hot-aqua extract of Tetradenia riparia leaves," IJPLS, vol. 5, no. 8, 2014.

[33] D. A. Santos and J. S. Hamdan, "Evaluation of Broth Microdilution Antifungal Susceptibility Testing Conditions for Trichophyton rubrum," Journal of Clinical Microbiology, vol. 43, no. 4, pp. 1917-1920, 2005.

[34] I. Ahmad and F. Aqil, "In vitro efficacy of bioactive extracts of 15 medicinal plants against ES $\beta$ L-producing multidrug-resistant enteric bacteria," Microbiological Research, vol. 162, no. 3, pp. 264-275, 2007.

[35] E. H. Endo, G. M. Costa, T. U. Nakamura, C. V. Nakamura, and B. P. Dias Filho, "Antidermatophytic activity of hydroalcoholic extracts from rosmarinus officinalis and tetradenia riparia," Journal de Mycologie Médicale, vol. 25, no. 4, pp. 274-279, 2015.

[36] H. Nowrozi, G. Nazeri, P. Adimi, M. Bashashati, and M. Emami, "Comparison of the activities of four antifungal agents in an in vitro model of dermatophyte nail infection "' Indian Journal of Dermatology, vol. 53, no. 3, p. 125, 2008.

[37] M. E. Barros, D. d. Santos, and J. S. Hamdan, "Evaluation of susceptibility of Trichophyton mentagrophytes and Trichophyton rubrum clinical isolates to antifungal drugs using a modified CLSI microdilution method (M38-A)," Journal of Medical Microbiology, vol. 56, no. 4, pp. 514-518, 2007. 


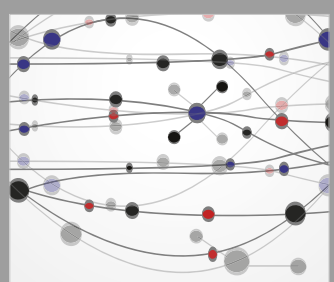

The Scientific World Journal
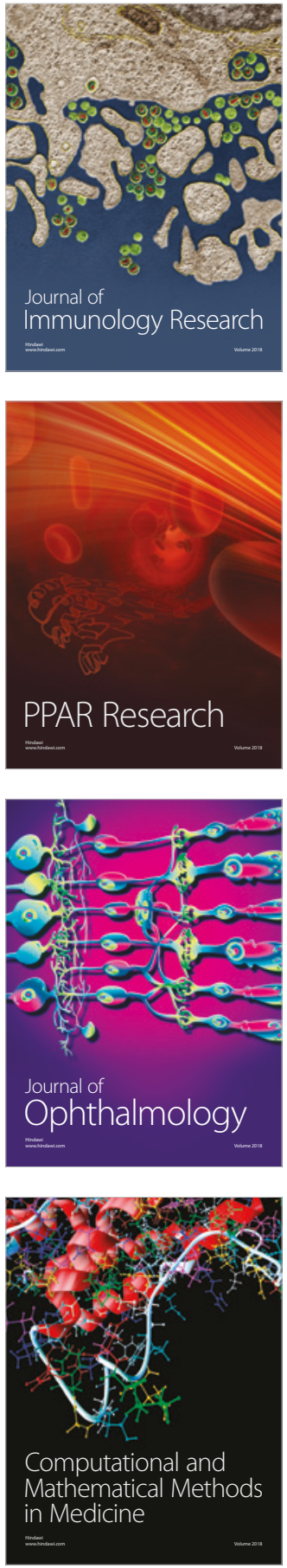

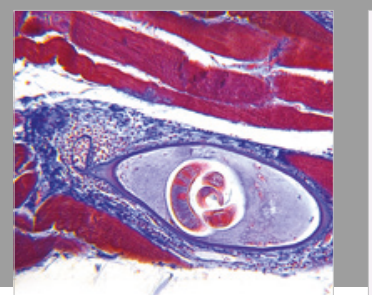

Gastroenterology Research and Practice

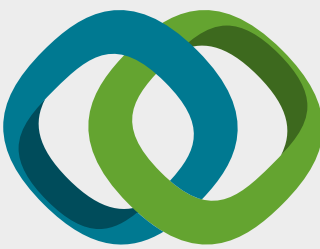

\section{Hindawi}

Submit your manuscripts at

www.hindawi.com
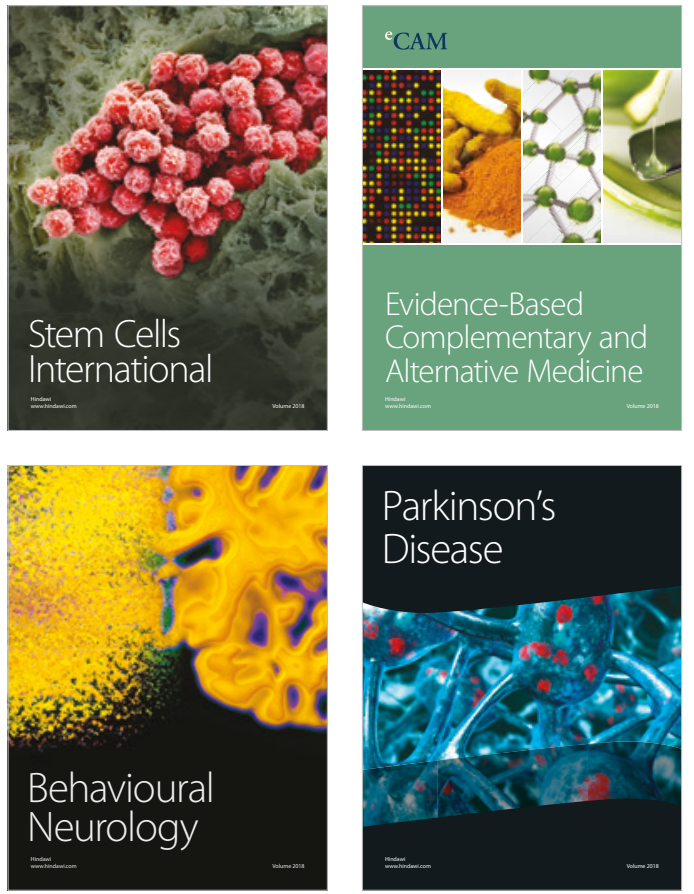

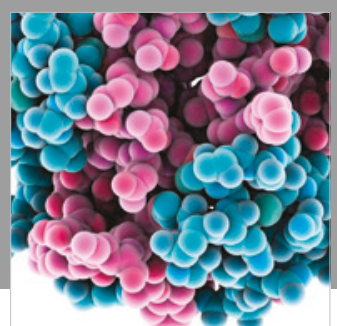

ournal of

Diabetes Research

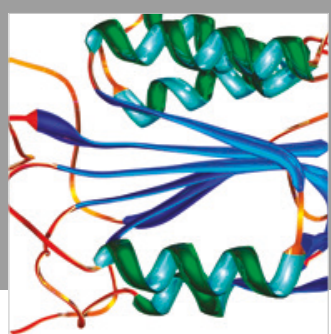

Disease Markers
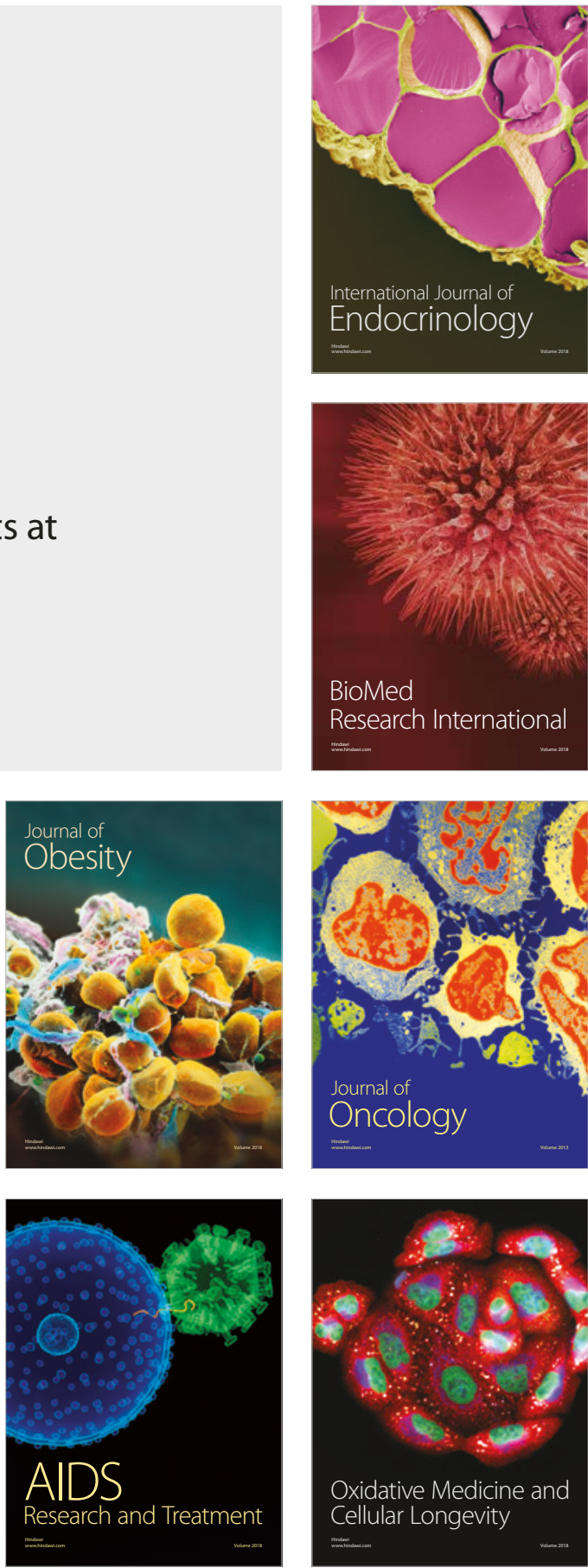